\title{
Robust $\mathcal{H}_{\infty}$ DIA Control of Levitated Steel Plates
}

\author{
Toru Namerikawa Member (Kanazawa University, toru@t.kanazawa-u.ac.jp) \\ Daisuke Mizutani Non-member (Nagaoka University of Technology, mizutani@stn.nagaokaut.ac.jp) \\ Shintaro Kuroki Non-member (Nagaoka University of Technology, shintaro@stn.nagaokaut.ac.jp)
}

Keywords: $\mathcal{H}_{\infty}$ DIA control, magnetic suspension system, robust control, levitated steel plate

This paper deals with an application of $\mathcal{H}_{\infty}$ DIA control to the magnetically levitated steel plates. The magnetically levitated steel plate technology is expected to prevent a surface quality of steel plate from deteriorating in a manufacturing process. The multiple steel plates levitation problem by a single controller has not been studied and the robust control system design for multiple plates are expected.

Our goal in this research is to suspend two thin steel plates stably by using four electromagnets without any physical contacts. We apply the robust $\mathcal{H}_{\infty}$ DIA control approach to the magnetically levitated steel plates. The $\mathcal{H}_{\infty}$ DIA control problem treats a mixed Disturbance and an Initial state uncertainty Attenuation and it is expected to provide a good transient property to a control system.

We have constructed an experimental system for levitated steel plates shown in Fig. 1. This system has five electromagnets and four of them are used in the feedback control in this research. Detail specification of two steel plates used in experiments is described in Table 1.

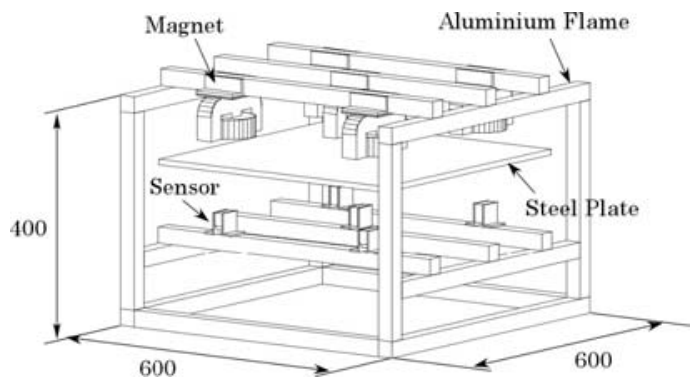

Fig. 1. Magnetically Levitated Steel Plates

A mathematical model of the system was derived where we assumed that the whole system can be divided to four independent subsystems. Four SISO control systems are designed individually by the $\mathcal{H}_{\infty}$ DIA control.

Then we set the generalized plant which contains design parameters for uncertainties and control performance, and designed an $\mathcal{H}_{\infty}$ DIA controller.

We conducted control experiments for two plates in Table 1 and evaluated control and robustness properties of the designed $\mathcal{H}_{\infty}$ DIA controller compared with the conventional PID controller. The
Table 1. Two Steel Plates

\begin{tabular}{|c|c|c|}
\hline & Plate 1 & Plate 2 \\
\hline Width & $500[\mathrm{~mm}]$ & $500[\mathrm{~mm}]$ \\
\hline Depth & $500[\mathrm{~mm}]$ & $500[\mathrm{~mm}]$ \\
\hline Thickness & $0.3[\mathrm{~mm}]$ & $0.5[\mathrm{~mm}]$ \\
\hline Mass & $0.537[\mathrm{~kg}]$ & $0.937[\mathrm{~kg}]$ \\
\hline
\end{tabular}

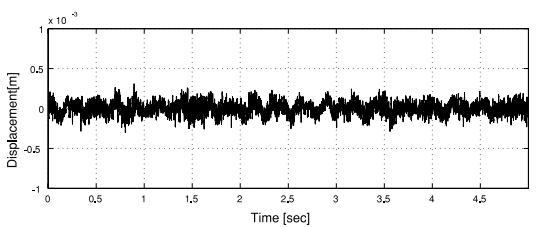

Fig. 2. $\mathcal{H}_{\infty}$ DIA: $0.3[\mathrm{~mm}]$

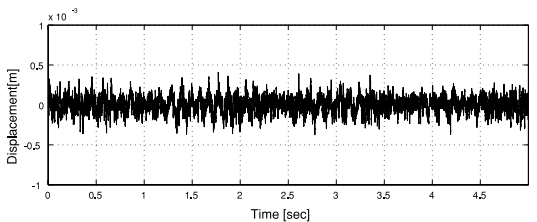

Fig. 3. PID: $0.3[\mathrm{~mm}]$

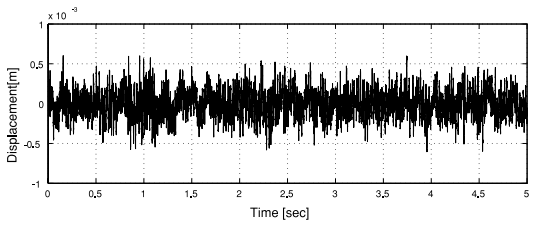

Fig. 4. $\mathcal{H}_{\infty}$ DIA: $0.5[\mathrm{~mm}]$

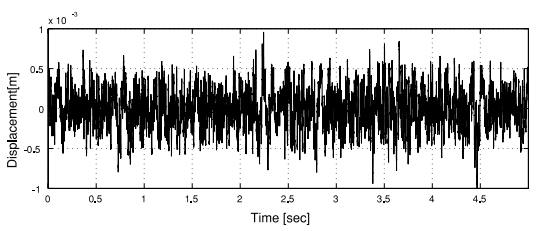

Fig. 5. PID: $0.5[\mathrm{~mm}]$

experimental results are shown in Figs. 2-5. They show that our proposed $\mathcal{H}_{\infty}$ DIA controller suppresses vibration of the steel plates and it has a robust stability for multiple steel plates. 


\title{
Robust $\mathcal{H}_{\infty}$ DIA Control of Levitated Steel Plates
}

\author{
Toru Namerikawa* Member \\ Daisuke Mizutani** $^{* *}$ Non-member \\ Shintaro Kuroki** $\quad$ Non-member
}

This paper deals with an application of $\mathcal{H}_{\infty}$ DIA control to the magnetically levitated steel plates. Our goal in this research is to suspend two thin steel plates stably by using four electromagnets without any physical contacts. We apply the robust $\mathcal{H}_{\infty}$ DIA control approach to the magnetically levitated steel plates. The $\mathcal{H}_{\infty}$ DIA control problem treats a mixed Disturbance and an Initial state uncertainty Attenuation and it is expected to provide a good transient property to a control system. Experimental results show that the proposed robust control approach is effective for suppressing an elasticity vibration of steel plates.

Keywords: $\mathcal{H}_{\infty}$ DIA control, magnetic suspension system, robust control, levitated steel plate

\section{Introduction}

The magnetically levitated steel plate technology is expected to prevent a surface quality of steel plate from deteriorating in a manufacturing process ${ }^{(1)(2)}$. This technology can also be applied to magnetic levitated transportation systems ${ }^{(3)}$.

In order to make this technology fit for practical use, a feedback controller should be able to suspend multiple steel plates, and to suppress an elasticity vibration of steel plates (4)(5).

There have been a lot of related works concerning magnetically levitated steel plate technology, e.g., steel plate transport systems, et al. The multiple steel plates levitation problem by a single controller, however, has not been studied and the robust control system design for multiple plates are expected.

Our goal in this research is to suspend two thin steel plates stably by using four electromagnets without any physical contacts. We apply the robust $\mathcal{H}_{\infty}$ DIA control approach ${ }^{(8)}$ to the magnetically levitated steel plates.

The $\mathcal{H}_{\infty}$ DIA control problem treats a mixed Disturbance and an Initial state uncertainty Attenuation and it is expected to provide a good transient property to the control system.

We have constructed an experimental system with four electromagnets for levitated steel plates. We drive a mathematical model of the system where we have assumed that the whole system can be divided to four independent subsystems.

Four SISO control systems are designed individually by the $\mathcal{H}_{\infty}$ DIA control theory. Then we set the generalized plant which contains design parameters for uncertainties and control performance, and design $\mathcal{H}_{\infty}$ DIA controllers for the

\footnotetext{
* Division of Electrical Engineering and Computer Science, Graduate School of Natural Science and Technology, Kanazawa University Kakuma, Kanazawa 920-1192

** Department of Mechanical Engineering, Nagaoka University of Technology

1603-1, Kamitomioka, Nagaoka 940-2188
}

generalized plant.

Finally we conduct control experiments for two plates and evaluate control and robustness properties of the designed $\mathcal{H}_{\infty}$ DIA controller compared with the conventional PID controller. The experimental results show that our proposed $\mathcal{H}_{\infty}$ DIA controller suppresses a vibration of the steel plates and it has a robust stability for multiple steel plates.

\section{Robust $\mathcal{H}_{\infty}$ DIA Control}

Consider the linear time-invariant system.

$$
\begin{aligned}
& \dot{x}=A x+B_{1} w+B_{2} u, \quad x(0)=x_{0} \\
& z=C_{1} x+D_{12} u \\
& y=C_{2} x+D_{21} w \ldots \ldots \ldots \ldots \ldots
\end{aligned}
$$

where $x \in R^{n}$ is the state and $x_{0}$ is the initial state; $u \in R^{r}$ is the control input; $y \in R^{m}$ is the observed output; $z \in R^{q}$ is the controlled output; $w \in R^{p}$ is the disturbance.

For system (1), a control $u(t)$ is given by linear timeinvariant system of the form

$$
\begin{aligned}
& u=J \zeta+K y \\
& \dot{\zeta}=G \zeta+H y, \quad \zeta(0)=0
\end{aligned}
$$

which makes the closed-loop system internally stable.

For the system and the controls described above, consider a mixed-attenuation problem as follows.

$\mathcal{H}_{\infty}$ DIA Control problem ${ }^{(8)}$

Find a control $u(t)$ attenuating disturbances and initial state uncertainties in the way that, for given $N>0, z$ satisfies

$$
\|z\|_{2}^{2}<\|w\|_{2}^{2}+x_{0}^{T} N^{-1} x_{0}
$$

for all $w \in L^{2}[0, \infty)$ and all $x_{0} \in R^{n}$, s.t., $\left(w, x_{0}\right) \neq 0$.

Such an admissible control is called the Disturbance and Initial state uncertainty Attenuation (DIA) control.

In order to solve the problem, we require the following three conditions.

(A1) There exists a solution $M>0$ to the Riccati equation 


$$
\begin{aligned}
& M\left(A-B_{2}\left(D_{12}^{T} D_{12}\right)^{-1} D_{12}^{T} C_{1}\right) \\
& \quad+\left(A-B_{2}\left(D_{12}^{T} D_{12}\right)^{-1} D_{12}^{T} C_{1}\right)^{T} M \\
& \quad-M\left(B_{2}\left(D_{12}^{T} D_{12}\right)^{-1} B_{2}^{T}-B_{1} B_{1}^{T}\right) M \\
& \quad+C_{1}^{T} C_{1}-C_{1}^{T} D_{12}\left(D_{12}^{T} D_{12}\right)^{-1} D_{12}^{T} C_{1}=0 \ldots \ldots \ldots
\end{aligned}
$$

s.t. $A-B_{2}\left(D_{12}^{T} D_{12}\right)^{-1} D_{12}^{T} C_{1}-B_{2}\left(D_{12}^{T} D_{12}\right)^{-1} B_{2}^{T} M+B_{1} B_{1}^{T} M$ is stable.

(A2) There exists a solution $P>0$ to the Riccati equation

$$
\begin{aligned}
(A & \left.-B_{1} D_{21}^{T}\left(D_{21} D_{21}^{T}\right)^{-1} C_{2}\right) P \\
& +P\left(A-B_{1} D_{21}^{T}\left(D_{21} D_{21}^{T}\right)^{-1} C_{2}\right)^{T} \\
& -P\left(C_{2}^{T}\left(D_{21} D_{21}^{T}\right)^{-1} C_{2}-C_{1}^{T} C_{1}\right) P \\
& +B_{1} B_{1}^{T}-B_{1} D_{21}^{T}\left(D_{21} D_{21}^{T}\right)^{-1} D_{21} B_{1}^{T}=0
\end{aligned}
$$

s.t. $A-B_{1} D_{21}^{T}\left(D_{21} D_{21}^{T}\right)^{-1} C_{2}-P C_{2}^{T}\left(D_{21} D_{21}^{T}\right)^{-1} C_{2}+P C_{1}^{T} C_{1}$ is stable.

\section{(A3) $\rho(P M)<1$}

where $\rho(X)$ denotes the spectral radius of matrix $X$. Then we obtained the following results.

Theorem $1^{(8)}$

Suppose that the conditions (A1), (A2), (A3) are satisfied. Then the $\mathcal{H}_{\infty}$ central control is given as

$$
\begin{aligned}
& u=-\left(D_{12}^{T} D_{12}\right)^{-1}\left(B_{2}^{T} M+D_{12}^{T} C_{1}\right)(I-P M)^{-1} \zeta \\
& \dot{\zeta}=A \zeta+B_{2} u+P C_{1}^{T}\left(C_{1} \zeta+D_{12} u\right) \\
& +\left(P C_{2}^{T}+B_{1} D_{21}^{T}\right)\left(D_{21} D_{21}^{T}\right)^{-1}\left(y-C_{2} \zeta\right) \\
& \zeta(0)=0
\end{aligned}
$$

The $\mathcal{H}_{\infty}$ central control (6) is a DIA control if and only if the condition (A4) is satisfied.

(A4) $Q+N^{-1}-P^{-1}>0$

where $Q$ is the maximal solution of the Riccati equation

$$
\begin{aligned}
& Q\left(A-B_{1} D_{21}^{T}\left(D_{21} D_{21}^{T}\right)^{-1} C_{2}\right. \\
& \left.\quad+\left(B_{1} B_{1}^{T}-B_{1} D_{21}^{T}\left(D_{21} D_{21}^{T}\right)^{-1} D_{21} B_{1}^{T}\right) P^{-1}\right) \\
& \quad+\left(A-B_{1} D_{21}^{T}\left(D_{21} D_{21}^{T}\right)^{-1} C_{2}\right. \\
& \left.\quad+\left(B_{1} B_{1}^{T}-B_{1} D_{21}^{T}\left(D_{21} D_{21}^{T}\right)^{-1} D_{21} B_{1}^{T}\right) P^{-1}\right)^{T} Q \\
& \quad-Q\left(B_{1}^{T}-D_{21}^{T}\left(D_{21} D_{21}^{T}\right)^{-1}\left(C_{2} P+D_{21} B_{1}^{T}\right) L\right)^{T} \\
& \quad \times\left(B_{1}^{T}-D_{21}^{T}\left(D_{21} D_{21}^{T}\right)^{-1}\left(C_{2} P+D_{21} B_{1}^{T}\right) L\right) Q \\
& =0 \ldots \ldots \ldots \ldots \ldots \ldots \ldots \ldots \ldots \ldots \ldots \ldots \ldots \ldots \ldots \ldots \ldots \ldots \ldots \ldots \ldots \ldots \ldots \ldots
\end{aligned}
$$

with $L:=(I-P M)^{-1}$.

\section{System Description and Modeling}

3.1 Construction We have constructed an experimental system for levitated steel plates shown in Fig. 1, and its model is shown schematically in Fig. 2.

This system has five electromagnets and four of them are used in the feedback control. Each electromagnet has its own optical gap sensor across the steel plate as shown in Fig. 3.

Detail specifications of two steel plates: a $0.3[\mathrm{~mm}]$ thick Steel Plate 1 (SP1) and a $0.5[\mathrm{~mm}]$ thick Steel Plate 2 (SP2)

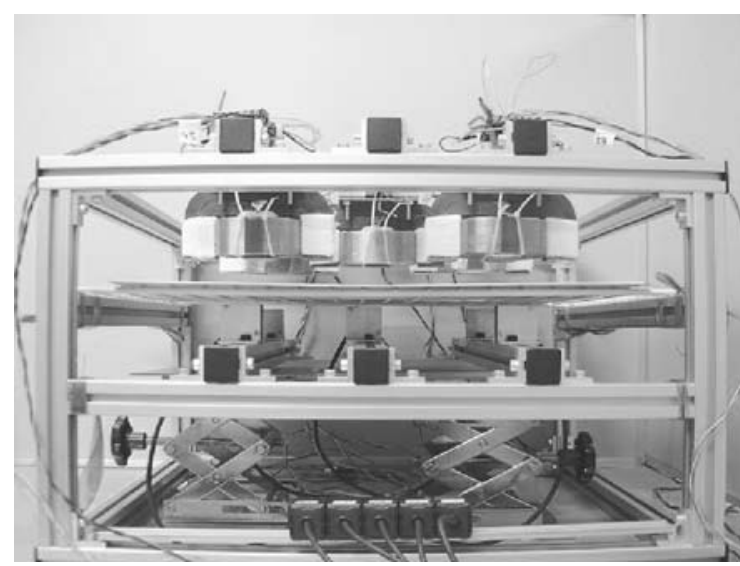

Fig. 1. Experimental setup

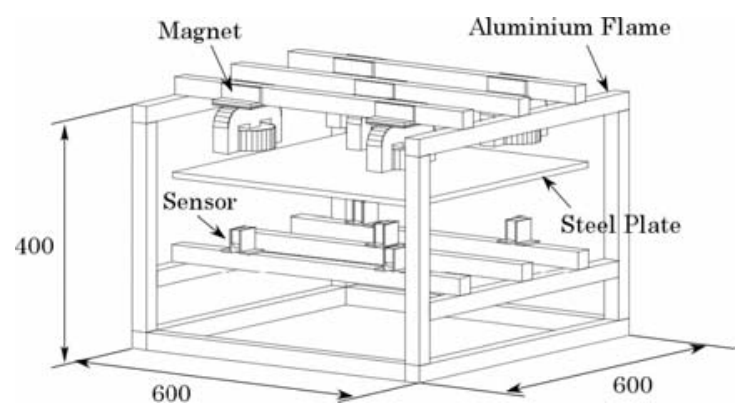

Fig. 2. System structure

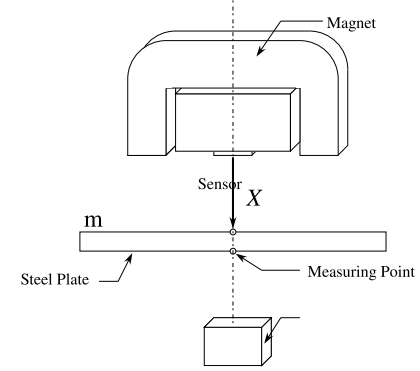

Fig. 3. Position relation between a electromagnet and a sensor

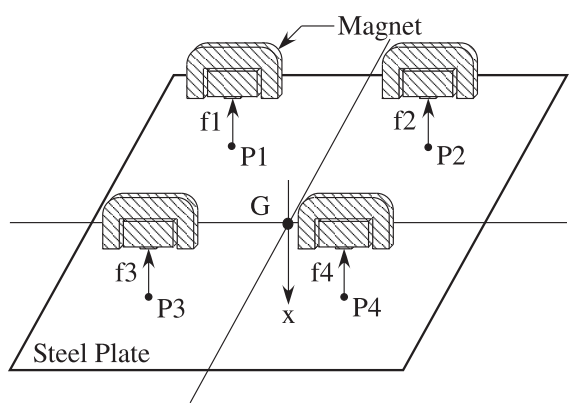

Fig. 4. Magnetically Levitated Steel Plates

used in experiments are described in Table 1 . Here the $S P l$ is a nominal suspended object and the $S P 2$ is a perturbed suspended object.

3.2 Mathematical Model In order to derive a model of the system by physical law, we introduce following assumptions.

- The whole system can be divided to four independent Single Input Single Output subsystems. 
Table 1. Specifications for the steel plates

\begin{tabular}{|l|c|c|}
\hline & Steel Plate 1 & Steel Plate 2 \\
\hline Wide & $500[\mathrm{~mm}]$ & $500[\mathrm{~mm}]$ \\
Depth & $500[\mathrm{~mm}]$ & $500[\mathrm{~mm}]$ \\
Thickness & $0.3[\mathrm{~mm}]$ & $0.5[\mathrm{~mm}]$ \\
Mass & $0.537[\mathrm{~kg}]$ & $0.937[\mathrm{~kg}]$ \\
\hline
\end{tabular}

Table 2. System Parameter

\begin{tabular}{|c|c|c|c|}
\hline Parameter & Symbol & $S P 1^{\text {a }}$ & $S P 2^{\mathrm{b}}$ \\
\hline $\begin{array}{l}\text { Mass of a quarter of } \\
\text { Steel Plate }\end{array}$ & $m[g]$ & 134 & 238 \\
\hline Steady Gap & $X[\mathrm{~m}]$ & $5.0 \times 10^{-3}$ & $5.0 \times 10^{-3}$ \\
\hline Coefficients of $f(t)$ & $k\left[\mathrm{Nm}^{2} / \mathrm{A}^{2}\right]$ & $8.50 \times 10^{-4}$ & $9.5 \times 10^{-4}$ \\
\hline position offset term & $x_{0}[\mathrm{~m}]$ & $2.80 \times 10^{-2}$ & $1.15 \times 10^{-3}$ \\
\hline Steady Current & $I[\mathrm{~A}]$ & 0.309 & 0.3595 \\
\hline
\end{tabular}

- Four electromagnets are identical (see Fig. 4).

Under these assumptions, we derive the equation of motion of the iron steel plate and the electromagnetic force equation as follows ${ }^{(9)}$.

$$
\begin{aligned}
& m \frac{d^{2} x(t)}{d t^{2}}=m g-f(t)+v_{m}(t) \\
& f(t)=k\left(\frac{I+i(t)}{X+x(t)+x_{0}}\right)^{2} \ldots
\end{aligned}
$$

where $m$ as the mass of the $1 / 4$ steel plate, $X$ as a steady gap between the electromagnet and the steel plate, $x(t)$ as a deviation from $X, I$ as a steady current, $i(t)$ as a deviation from $I$, $f(t)$ as an electromagnet force, $k, x_{0}$ are coefficients of $f(t)$, $v_{m}(t)$ as a disturbance force.

The electromagnetic force (9) is linearized around the operating point by the Taylor series expansion as

$$
\begin{aligned}
& f(t)=k\left(\frac{I}{X+x_{0}}\right)^{2}-K_{x} x(t)+K_{i} i(t) \\
& K_{x}=\frac{2 k I^{2}}{\left(X+x_{0}\right)^{3}}, K_{i}=\frac{2 k I}{\left(X+x_{0}\right)^{2}}
\end{aligned}
$$

The sensor provides the information for the gap $x(t)$. Hence the measurement equation can be written as

$$
y_{g}(t)=x(t)+w_{0}(t)
$$

where $w_{0}(t)$ represents the sensor noise as well as the model uncertainties.

Thus, summing up the above results, the state equations for the system ${ }^{(9)}$ are

$$
\begin{aligned}
& \dot{x}_{g}=A_{g} x_{g}+B_{g} u_{g}+D_{g} v_{0} \ldots \ldots \ldots \ldots \ldots \\
& y_{g}=C_{g} x_{g}+w_{0} \\
& x_{g}:=\left[\begin{array}{ll}
x & \dot{x}
\end{array}\right]^{T}, u_{g}:=i, v_{0}:=v_{m} \\
& A_{g}=\left[\begin{array}{cc}
0 & 1 \\
K_{x} & 0
\end{array}\right], B_{g}\left[\begin{array}{c}
0 \\
-K_{i}
\end{array}\right], D_{g}=\left[\begin{array}{c}
0 \\
1 / m
\end{array}\right] \\
& C_{g}=\left[\begin{array}{ll}
1 & 0
\end{array}\right]
\end{aligned}
$$

\section{Control System Design}

Let $v_{0}$ and $w_{0}$ be of the form with the frequency weighting functions $W_{v}$ and $W_{w}$ as

$$
v_{0}=W_{v}(s) w_{2}
$$

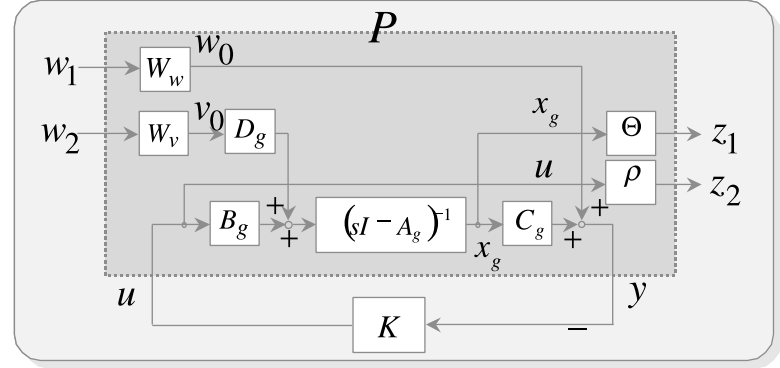

Fig. 5. Generalized Plant

$$
\begin{aligned}
W_{v} & =\Phi C_{v}\left(s I-A_{v}\right)^{-1} B_{v}, \Phi=\left[\begin{array}{ll}
1 & 1
\end{array}\right]^{T} \\
w_{0} & =W_{w} w_{1} . \cdots \cdots \cdots \cdots \cdots \ldots \ldots \ldots \ldots \ldots \ldots \ldots \ldots
\end{aligned}
$$

The gap $x(t)$ and the corresponding velocity $\dot{x}(t)$ are restricted by the weight $\Theta$, and the control input $u(t)$ also should be regulated to suppress the energy, where its weight is expressed by $\rho$; i.e.,

$$
\begin{aligned}
& z_{1}=\Theta x_{g}, \quad \Theta=\operatorname{diag}\left[\begin{array}{ll}
\theta_{1} & \theta_{2}
\end{array}\right] . \\
& z_{2}=\rho u_{g} \ldots \ldots \ldots \ldots \ldots \ldots \ldots \ldots \ldots \ldots \ldots \ldots \ldots \ldots \ldots
\end{aligned}
$$

Finally, we can construct the generalized plant (17) in the following;

$$
\begin{aligned}
& \dot{x}=A x+B_{1} w+B_{2} u \\
& z=C_{1} x+D_{12} u \\
& y=C_{2} x+D_{21} w \ldots \ldots \ldots \ldots \ldots \ldots \ldots \ldots \ldots \ldots \ldots \\
& A=\left[\begin{array}{cc}
A_{g} & D_{g} C_{w} \\
0 & A_{w}
\end{array}\right], B_{1}=\left[\begin{array}{cc}
0 & D_{g} D_{w} \\
0 & B_{w}
\end{array}\right] \\
& B_{2}=\left[\begin{array}{c}
B_{g} \\
0
\end{array}\right], C_{1}=\left[\begin{array}{cc}
\Theta & 0 \\
0 & 0
\end{array}\right], D_{12}=\left[\begin{array}{l}
0 \\
\rho
\end{array}\right] \\
& C_{2}=\left[\begin{array}{ll}
C_{g} & 0
\end{array}\right], D_{21}=\left[\begin{array}{ll}
W_{w} & 0
\end{array}\right]
\end{aligned}
$$

where $x:=\left[\begin{array}{ll}x_{g}^{T} & x_{v}^{T}\end{array}\right]^{T}$ and $x_{v}$ is a state of $W_{v}(s), w:=\left[\begin{array}{ll}w_{1}^{T} & w_{2}^{T}\end{array}\right]^{T}$ and $z:=\left[\begin{array}{ll}z_{1}^{T} & z_{2}^{T}\end{array}\right]^{T}$.

Now our control problem setup is as followed.

Control Problem Setup: Finding an admissible controller $K(s)$ that attenuates disturbance and initial state uncertainties to achieve DIA condition in (3).

4.1 $\mathcal{H}_{\infty}$ DIA Controller After some control system design iterations, the final parameter of the $\mathcal{H}_{\infty}$ DIA Controller was chosen as follows.

$$
\begin{aligned}
& W_{v}=\frac{5 \times 10^{3}}{s+1.0 \times 10^{-2}} \\
& W_{w}=5 \times 10^{-1} \\
& \Theta=\operatorname{diag}\left[\begin{array}{ll}
1 \times 10^{-2} & 1 \times 10^{-4}
\end{array}\right] \\
& \rho=1 \times 10^{-6} \ldots \ldots \ldots \ldots \ldots \ldots .
\end{aligned}
$$

These design parameters give the $\mathcal{H}_{\infty}$ DIA controller as follows, where the maximum value of the weighting matrix $N$ is $N=1.013 \times 10^{-2}$.

$$
K_{D I A}(s)=\frac{1.96 \times 10^{6}(s+7.48)(s+49.3)}{(s+6250)(s+224)\left(s+9.78 \times 10^{-3}\right)}
$$

The frequency response of the $\mathcal{H}_{\infty}$ DIA controller $K_{D I A}$ is shown in Fig. 6 by a solid line. 


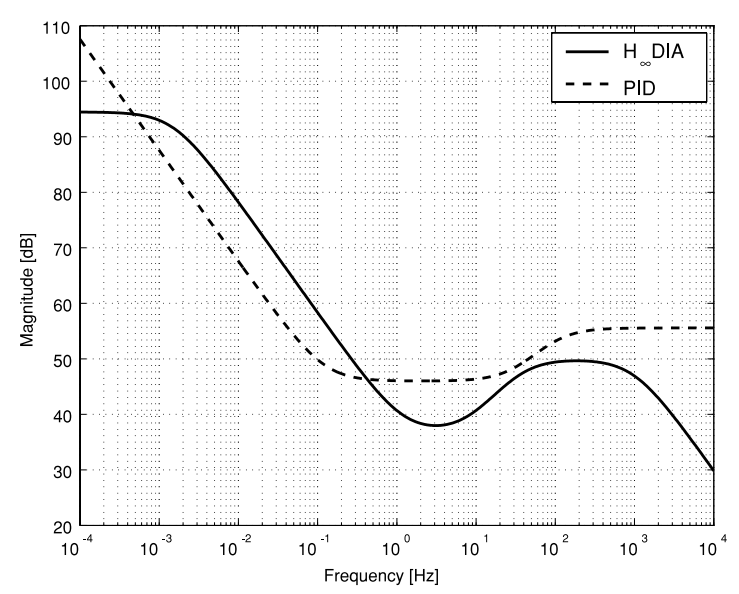

Fig. 6. Frequency Responses of Two Controllers

4.2 PID Controller We designed a saturated PID controller for the comparison with the proposed $\mathcal{H}_{\infty}$ DIA controller. In order to suppress a vibration of the closed-loop system at the high frequency, a gain of the PID controller is saturated at around $100[\mathrm{~Hz}]$. The transfer function of the final saturated PID controller $K_{P I D}(s)$ is as follows.

$$
K_{P I D}(s)=\frac{K_{D} s^{2}+K_{P} s+K_{I}}{s\left(1+\frac{K_{D} / K_{P}}{N} s\right)}
$$

where

$$
K_{P}=200, K_{I}=150, K_{D}=1.0, N=3
$$

The frequency response of the controller $K_{P I D}(s)$ is also shown in Fig. 6 by a dashed line.

\section{Experimental Evaluation}

We conducted experiments to evaluate properties of the $\mathcal{H}_{\infty}$ DIA controller for steel plate levitation system.

All experimental results shown below are time responses at the electromagnet 1 at $\mathrm{P} 1$ in Fig. 4. The other responses at P2-P4 show similar to them at P1.

5.1 Nominal Stability and Performance First we conducted experiments by using the nominal steel plate $S P 1$. Steady state position responses for stability evaluation of $\mathcal{H}_{\infty}$ DIA controller and PID controller are shown in Figs. 7 and 13 , respectively. These figures show that $\mathcal{H}_{\infty}$ DIA control suppress a vibration of the steel plate compared with PID.

Transient step responses for performance evaluation of both controllers are shown in Figs. 8 and 14, respectively. $\mathcal{H}_{\infty}$ DIA shows relatively bigger overshoot but convergence of the controller is better and the steady state vibration is suppressed.

Figs. 9 and 15 are time responses for step disturbance whose magnitude is about 20 [\%] of the steady-state electromagnetic force. $\mathcal{H}_{\infty}$ DIA shows also relatively bigger overshoot but the steady state vibration is suppressed.

5.2 Robustness for multiple plates Robust stability was checked by using the steel plate $S P 2$. Steady state response of $\mathcal{H}_{\infty}$ DIA control and PID are shown in Figs.10 and 16 , respectively. Both controllers achieve robust stability and the closed-loop system with both controllers are stable for SP2.
Table 3. Vibration of center of Steel Plate in Steady State

\begin{tabular}{|c|c||c|c|c|}
\hline \multicolumn{2}{|c|}{} & $S P 1$ & $S P 2$ & $\begin{array}{c}\text { Increasing } \\
\text { Rate }\end{array}$ \\
\hline \hline \multirow{2}{*}{$\mathcal{H}_{\infty}$ DIA } & $\begin{array}{c}\text { Maximum } \\
\text { Amplitude } \\
{[\mathrm{mm}]}\end{array}$ & 0.2 & 0.4 & $+100[\%]$ \\
\cline { 2 - 5 } & $\begin{array}{c}\text { RMS of } \\
\text { vibration } \\
\text { [mm] }\end{array}$ & 0.083 & 0.180 & $+118[\%]$ \\
\hline \hline \multirow{3}{*}{ PID } & $\begin{array}{c}\text { Maximum } \\
\text { Amplitude } \\
{[\mathrm{mm}]}\end{array}$ & 0.3 & 0.8 & $+167[\%]$ \\
\cline { 2 - 5 } & $\begin{array}{c}\text { RMS of } \\
\text { vibration } \\
{[\mathrm{mm}]}\end{array}$ & 0.117 & 0.312 & $+167[\%]$ \\
\hline
\end{tabular}

Our proposed $\mathcal{H}_{\infty}$ DIA controller suppresses a vibration of the steel plates and it has a robust stability for multiple steel plates. $\mathcal{H}_{\infty}$ DIA control restrains a deterioration and a vibration compared with PID controller.

Transient step responses of both controllers are shown in Figs. 11 and 17, respectively. Disturbance responses of both controllers are also shown in Figs. 9 and 15.

These are similar to results in the nominal case and the $\mathcal{H}_{\infty}$ DIA controller suppresses the steady state vibration.

The maximum amplitude of the vibration of time responses and their root mean square value at the steady state in 5 [s] are summarized in Table 3.

Table 3 shows that the $\mathcal{H}_{\infty}$ DIA controller suppresses the steady state vibration and a deterioration caused by the model perturbation is reduced by the robust $\mathcal{H}_{\infty}$ DIA controller.

\section{Conclusion}

This paper dealt with an application of the robust $\mathcal{H}_{\infty}$ DIA control to the magnetically levitated steel plates.

Our goal in this research was to suspend two thin steel plates $S P 1$ and $S P 2$ stably by using four electromagnets. We applied the robust $\mathcal{H}_{\infty}$ DIA control approach ${ }^{(8)}$ to the magnetically levitated steel plates.

We have constructed an experimental system with four electromagnets for levitated steel plates. We derived a mathematical model of the system where we assumed that the whole system could be divided to four independent subsystems. Four SISO control systems were designed individually by the $\mathcal{H}_{\infty}$ DIA control theory. Then we set the generalized plant which contains design parameters for uncertainties and control performance, and designed $\mathcal{H}_{\infty}$ DIA controllers for the generalized plant.

Finally we conducted several control experiments for two plates and evaluated control and robustness properties of the designed $\mathcal{H}_{\infty}$ DIA controller compared with the conventional PID controller. The experimental results show that our proposed $\mathcal{H}_{\infty}$ DIA controller suppressed a vibration of the steel plates in the steady state and it had a robust stability for multiple steel plates.

Next goal is a stable suspension of 1 [mm] thick steel plate without any physical contact by using $\mathcal{H}_{\infty}$ DIA control and Multi-Input and Multi-Output system representation of the controlled system. 


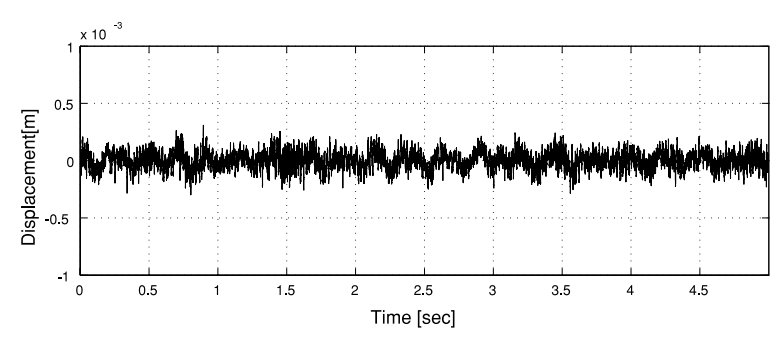

Fig. 7. Steady state of $S P 1$ for $\mathcal{H}_{\infty}$ DIA

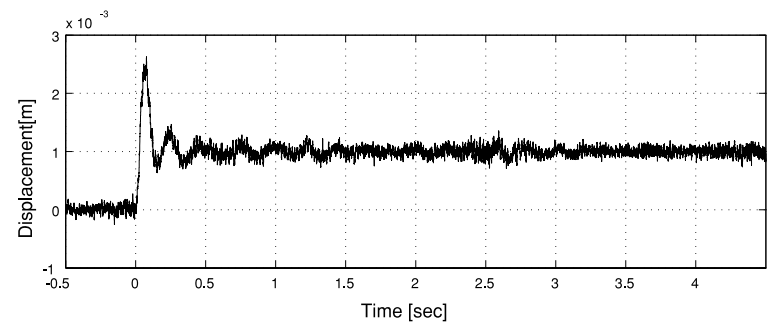

Fig. 8. Step response of $S P 1$ for $\mathcal{H}_{\infty}$ DIA

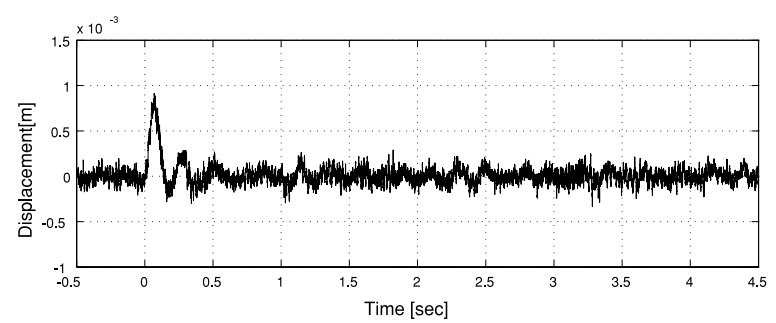

Fig. 9. Disturbance response of $S P 1$ for $\mathcal{H}_{\infty}$ DIA

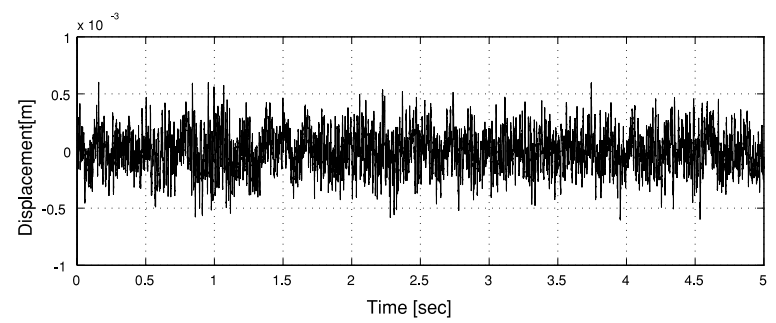

Fig. 10. Steady state of $S P 2$ for $\mathcal{H}_{\infty}$ DIA

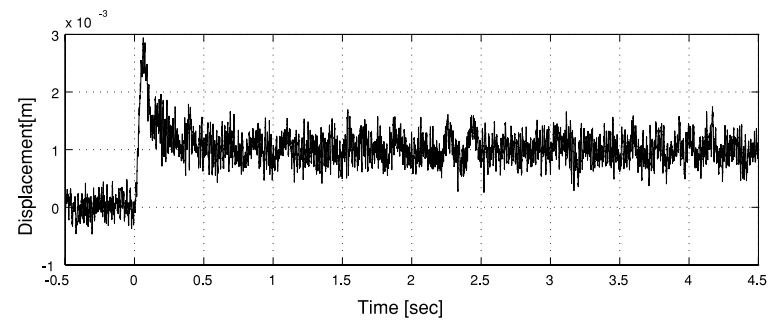

Fig. 11. Step response of $S P 2$ for $\mathcal{H}_{\infty}$ DIA

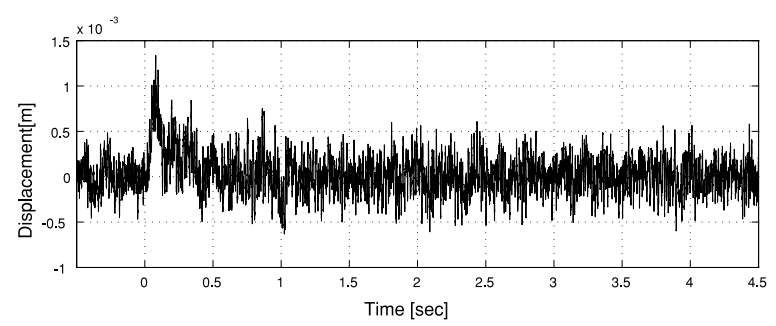

Fig. 12. Disturbance response of $S P 2$ for $\mathcal{H}_{\infty}$ DIA

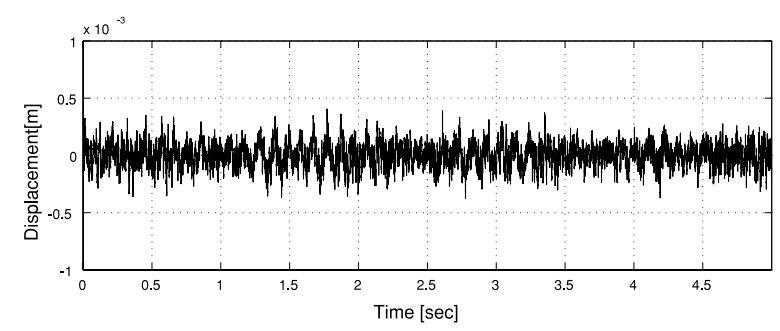

Fig. 13. Steady state of $S P 1$ for PID

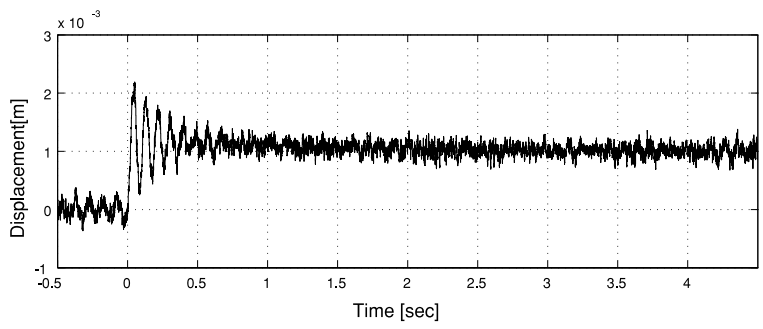

Fig. 14. Step response of $S P 1$ for PID

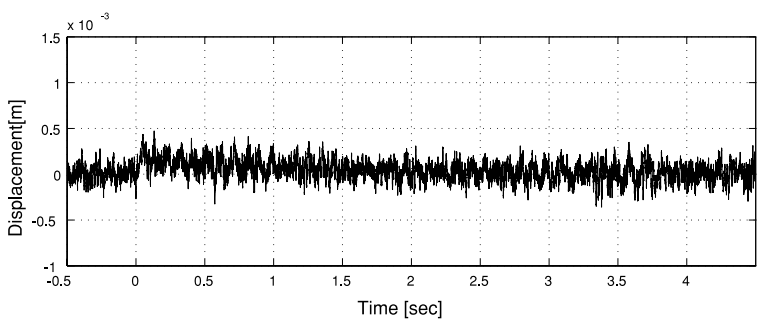

Fig. 15. Disturbance response of $S P 1$ for PID

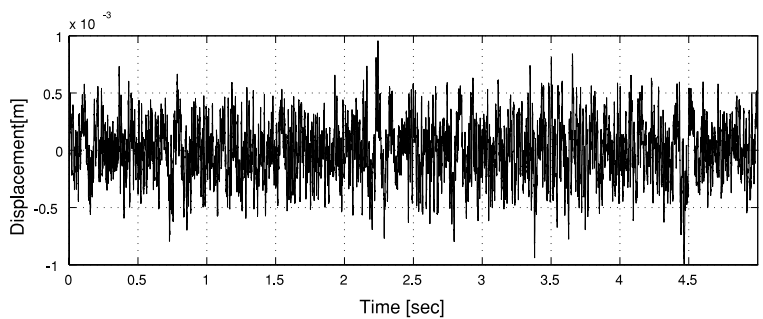

Fig. 16. Steady state of $S P 2$ for PID

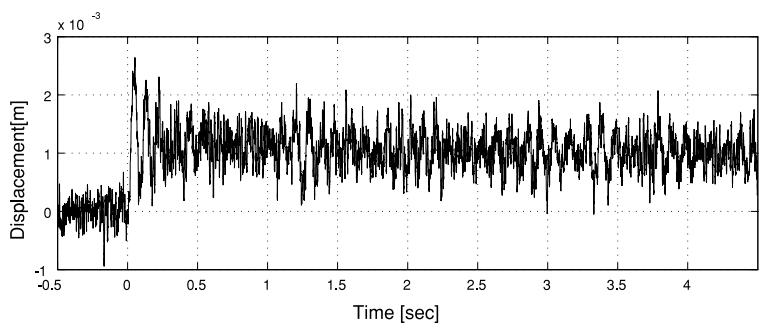

Fig. 17. Step response of $S P 2$ for PID

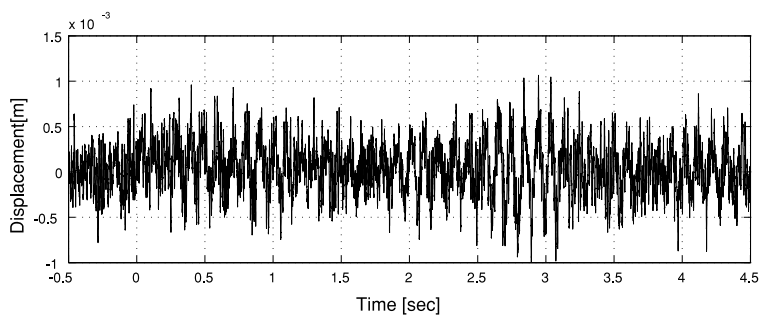

Fig. 18. Disturbance response of $S P 2$ for PID 
(Manuscript received Dec. 19, 2005,

revised April 10, 2006)

\section{References}

( 1 ) T. Nakagawa and M. Hama: "Study of Magnetic Levitation Control by Means of Correcting Gap Length Command for a Thin Steel Plate", T. IEE Japan, Vol.120-D, No.4, pp.489-494 (2000) (in Japanese)

( 2 ) M. Morishita and M. Akashi: "Inclination Guide Experiment for Magnetically Levitated Steel Plates", 1988 National Convention Record, IEEJ, pp.5183 (1998) (in Japanese)

( 3 ) H. Gurol and R.W. Baldi: "General Atomics Urban Maglev Test Track Status", Proc. of The Fifth International Symp. on Linear Drives for Industry Applications, pp.251-253 (2005)

( 4 ) Y. Oshinoya, K. Ishibashi, and T. Sekihara: "Conveyance Control for an Electromagnetic Levitation Rectangular Sheet Steel (Proposition of Noncontact Control Mechanism for the Horizontal Inertial Force of the Steel Plate which Suppressed the Elastic Vibration)", Trans. on JSME. Series C, Vol.68, No.669, pp.86-92 (2002) (in Japanese)

( 5 ) T. Ishiwatari, M. Watada, S. Torii, and D. Ebihara: "Effect of suppressing vibration on thin steel sheet suspended by magnetic levitation control which includes two desired values", Technical Meeting on Linear Drives, IEEJ, LD98-37, pp.43-48 (1998) (in Japanese)

( 6 ) K. Tada and S. Torii: "The Design Manual of Voltage-Controlled and Current-Controlled Observer in Sensorless Magnetic Levitation System of Steel Plate", Proc. of The Fifth International Symp. on Linear Drives for Industry Applications, pp.433-436 (2005)

( 7 ) M. Castelli, M. Martinez-Iturralde, G. Martinez, A. Garcia Rico, and J. Florez: "Linear Induction Motor Control for Simultaneous Propulsion and Levitation of an Aluminium Plate", Proc. of The Fifth International Symp. on Linear Drives for Industry Applications, pp.465-4468 (2005)

( 8 ) T. Namerikawa, M. Fujita, R.S. Smith, and K. Uchida: "On the $\mathcal{H}_{\infty}$ Control System Design Attenuating Initial State Uncertainties", Trans. on SICE, Vol.40, No.3, pp.307-314 (2004)

( 9 ) M. Fujita, T. Namerikawa, F. Matsumuta, and K. Uchida: " $\mu$-Synthesis of an Electromagnetic Suspension System", IEEE Trans. on Automatic Control, Vol.40, No.3, pp.530-536 (1995)
Toru Namerikawa (Member) received the B.E., M.E. and Dr. of En-

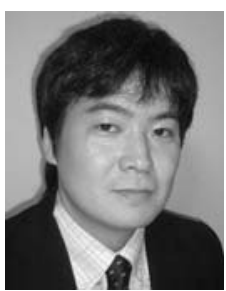
gineering degrees in Electrical and Computer Engineering from Kanazawa University, Japan, in 1991, 1993 and 1997, respectively. From 1994 until 2002, he was with Kanazawa University as a Research Associate. From 2002 until 2005, he was with Nagaoka University of Technology as an Associate Professor. In January 2006, he joined Kanazawa University, where he is currently an Associate Professor at Division of Electrical Engineering and Computer Science, Graduate School of Natural Science and Technology. He has held visiting positions at: the Swiss Federal Institute of Technology, Zurich, Switzerland in 1998; the University of California, Santa Barbara from 2001 until 2002. His research interests are robust control and its applications to electro-mechanical systems including magnetic bearings.

Daisuke Mizutani (Non-member) was born in Mie, Japan, on Jan-

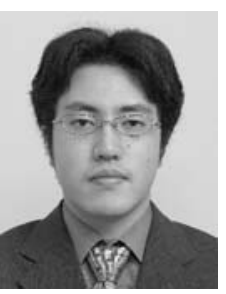
uary 1981. He received a BS degree in 2003 and MS degree in 2005 from Nagaoka University of Technology. He is presently at FUJI MACHINE MFG.CO., LTD.

Shintaro Kuroki (Non-member) was born in Osaka, Japan, on

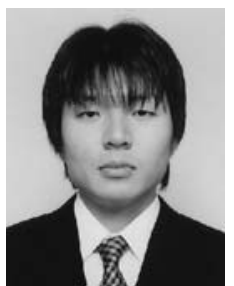
November 1982. He received a BS degree Nagaoka University of Technology in 2005 . He is presently a student of master's course at Nagaoka University of Technology and has studied on fault-tolerant system of levitated steel plates. 